\title{
Mechanical Bowel Preparation, Do It or Not: When Crossing a River, What Do People Do?
}

\author{
Hungdai Kim \\ Department of Surgery, Gastrointestinal Cancer Center, Kangbuk Samsung Hospital, Seoul, Korea
}

\section{See Article on Page 106-111}

At a river crossing, people usually prefer taking a bridge to swimming. However, if the bridge seems not to be strong enough, what would they do?

The authors conducted a retrospective study to demonstrate the usefulness of intraoperative bowel decompression and irrigation in patients with left colonic obstruction or left colon perforation [1]. They used a new intraoperative colonic irrigator (NICI, MITech, Seoul, Korea) for bowel irrigation. Their results support the safety and feasibility of intraoperative colonic irrigation and primary anastomosis. According to a systematic review of intraoperative colonic irrigation vs. manual decompression, manual decompression was comparable to colonic irrigation for primary anastomosis in obstructed left-sided colorectal emergencies, with no significant increase in mortality, leak or infection rate. Thus, the authors might conduct a further comparative study to prove the superiority of intracolonic bowel preparation [2].

We should take a more fundamental approach to this issue. Significant debate exists about the role of mechanical bowel preparation (MBP) itself. According to articles mainly from Europe, MBP before elective colorectal surgery can safely be abandoned. Contant et al. [3] conducted a multicenter, randomized, noninferiority study to compare the rates of anastomotic leakage after elective colorectal resections and primary anastomoses between patients who did or did not have MBP. They found that the rate of anastomotic leakage did not differ between the 2 groups ( $4.8 \%$ vs. $5.4 \%$, $\mathrm{P}=0.69$ ). Ciga et al. [4] compared one-stage colectomies of the descending colon without mechanical preparation in emergency

Correspondence to: Hungdai Kim, M.D.

Department of Surgery, Gastrointestinal Cancer Center, Kangbuk Samsung

Hospital, 29 Saemunan-ro, Jongno-gu, Seoul 31116, Korea

Tel: +82-2-2001-8541, Fax: +82-2-2001-2131

E-mail: hungdai.kim@samsung.com

(c) 2017 The Korean Society of Coloproctology

This is an open-access article distributed under the terms of the Creative Commons Attribution NonCommercial License (http://creativecommons.org/licenses/by-nc/4.0) which permits unrestricted noncommercial use, distribution, and reproduction in any medium, provided the original work is properly cited. and elective surgery. No differences were found in mortality, anastomotic dehiscence, or surgical site infection between the 2 groups. Thus, they concluded that in selected patients, an emergency left-colon, 1-stage resection and primary anastomosis might be performed without colonic preparation as safely as elective surgery. On the other hand, Rencuzogullari et al. [5] recently reported the database review of the American College of Surgeons National Surgical Quality Improvement Program to determine predictors of anastomotic leak in elderly patients undergoing a colectomy. They found that the omission of MBP was deleterious in terms of surgical site infection, anastomotic dehiscence, ileus, length of hospitalization, and even recurrence of the neoplasm when compared to colon surgery with MBP, especially when (in the American studies) MBP was combined with oral antibiotic administration.

The no MBP group presents several pieces of rational evidence. Firstly, MBP does not minimize the risk of postoperative septic complications, such as anastomotic dehiscence and superficial surgical site infections. Secondly, MBP is a time-consuming, expensive procedure and causes severe discomfort to the patient. Lastly, MBP has been associated with serious complications, such as electrolyte and volume disturbances, in both healthy patients and patients with existing cardiac or renal disease. Furthermore they pointed out that no prospective, randomized, controlled study supporting MBP as an efficient tool against leakage and infectious has been published. Yes, all of their conclusions are, of course, true. However, I cannot help but worry that they saw only what they wanted to see.

What are we missing in our practice? This issue includes a nonscientific problem. That is, health care providers should not forget that the safety of patients is the most important and invariant virtue in medical practice. Thus, we must consider the safety of patients before cost effectiveness or shortness of hospital stay. Frankly speaking, everybody knows every anastomosis, however good, has a risk of leakage, and everybody knows that the clinical course of fecal peritonitis is worse than that of any other type of peritonitis. The mortality rate from generalized fecal peritonitis varies from center to center, but studies have reported mortality rates in excess of 50\% [6]. This means that albeit the incidence of 
severe complication being very low, if a complication happens, the influence on the patient may be too serious to overcome. Every health care provider is directly responsible for protecting patients from such hazardous conditions. Must we sacrifice a few for the benefit of many?

In my personal opinion, intraoperative bowel irrigation should be performed regardless of data significance. This is not a matter of the usefulness of the procedure; it is the matter of the dignity of the patient. If I am driving a car alone and come to a bridge of uncertain durability, I may cross that bridge. However, if I am driving a school bus with full of children, I will take a detour without hesitation.

\section{CONFLICT OF INTEREST}

No potential conflict of interest relevant to this article was reported.

\section{REFERENCES}

1. Hong Y, Nam S, Kang JG. The usefulness of intraoperative colonic irrigation and primary anastomosis in patients requiring a left colon resection. Ann Coloproctol 2017;33:106-11.

2. Kam MH, Tang CL, Chan E, Lim JF, Eu KW. Systematic review of intraoperative colonic irrigation vs. manual decompression in obstructed left-sided colorectal emergencies. Int J Colorectal Dis 2009;24:1031-7.

3. Contant CM, Hop WC, van't Sant HP, Oostvogel HJ, Smeets HJ, Stassen LP, et al. Mechanical bowel preparation for elective colorectal surgery: a multicentre randomised trial. Lancet 2007; 370:2112-7.

4. Ciga MA, Oteiza F, Fernández L, de Miguel M, Ortiz H. Comparative study of one-stage colectomy of the descending colon in emergency and elective surgery without mechanical preparation. Dis Colon Rectum 2010;53:1524-9.

5. Rencuzogullari A, Benlice C, Valente M, Abbas MA, Remzi FH, Gorgun E. Predictors of anastomotic leak in elderly patients after colectomy: nomogram-based assessment From the American College of Surgeons National Surgical Quality Program Procedure-Targeted Cohort. Dis Colon Rectum 2017;60:527-36.

6. Vermeulen J, Gosselink MP, Hop WC, Lange JF, Coene PP, van der Harst E, et al. Hospital mortality after emergency surgery for perforated diverticulitis. Ned Tijdschr Geneeskd 2009;153:120914. 